\title{
Learning Analytics: Richer Perspectives Across Stakeholders
}

\author{
Simon Knight, Shane Dawson, Dragan Gašević, Jelena Jovanović, Arnon Hershkovitz \\ Editors, Journal of Learning Analytics \\ jla.editorial@gmail.com
}

\begin{abstract}
This issue of the Journal of Learning Analytics features seven research papers, complemented by a practitioner research paper (Dvorak \& Jia). Papers by McCoy and Shih, and Knight, Brozina, and Novoselich discuss the important topic of educators working with educational data, alongside (in the latter paper) student perspectives on learning analytics. Douglas, Bermel, Alam, and Madhavan; and Waddington, Nam, Lonn, and Teasley offer empirical insight on developing a richer perspective on learning material interaction and engagement in online learning contexts (MOOCs, and LMS' respectively). Dvorak and Jia bring a practitioner perspective to the issue in their discussion of approaches to analyzing online work habits via timeliness, regularity, and intensity. Sutherland and White, and Vieira, Goldstein, Purzer, and Magana offer focus on specific subject-based learning activities (algebra learning, and student experimentation strategies in engineering design, respectively). Finally, Howley and Rosé discuss the complex interactions of theory and method in computational modeling of group learning processes. The issue also features a special section on learning analytics tutorials, edited by Gašević and Pechenizkiy. The editorial concludes with a report of the recent 'hot spots section' consultation from the editorial team of the journal.
\end{abstract}

Keywords: learning analytics, tutorials, MOOCs, early warning systems, learning management systems, practitioners, predictive analytics

\section{DEVELOPING UNDERSTANDING OF LEARNING ANALYTICS ACROSS STAKEHOLDERS}

For learning analytics to maximise its impact, we must better understand the learning contexts from which our educational data are derived, and how such analytics are deployed in teaching practice. By unlocking and understanding the perspectives of the vast range of learning analytics stakeholders we can begin to establish an ontology that can better describe and frame the learning data and the types of analyses and feedback that can optimise learning and teaching processes. Simply put, analytics cannot achieve its potential without practitioners embedding them into pedagogic contexts (Clow, 2012), and understanding how such analytics can aid their student learning. Furthermore, learners require the access to and capacity to interpret analytics in the context of their own learning and sensemaking (Wise, Vytasek, Hausknecht, \& Zhao, 2016). The process of engaging stakeholders and the provision of transparent data and analytics to end-users are important, but they are only one part of a larger, more complex challenge. For instance, there may be differences, in approaches required in subject domains; 
(2016). Learning analytics: Richer perspectives across stakeholders. Journal of Learning Analytics, 3(3), 1-4. http://dx.doi.org/10.18608/jla.2016.33.1

student cohorts; cultural aspects as well as individual student perspectives on the use and application of such data (McPherson, Tong, Fatt, \& Liu, 2016).

In the present issue, McCoy and Shih, and Knight, Brozina, and Novoselich explore these challenges, discussing the experiences of educators working with educational data, alongside (in the latter paper) student perspectives on learning analytics. McCoy and Shih's paper discusses "teachers as producers and not just consumers of data analytics", noting the potential and barriers in teacher use of educational data. Knight, Brozina, and Novoselich take a similar approach, discussing practices "that select relevant data and develop models with learners and teachers instead of for learners and teachers...", in the specific context of first-year engineering undergraduates and their instructors' perspectives on the potential of learning analytics.

\section{DEVELOPING RICHER ENGAGEMENT MEASURES}

The development of richer models of learning engagement are discussed from a different perspective by Douglas, Bermel, Alam, and Madhavan, and Waddington, Nam, Lonn, and Teasley both of whom argue for moving beyond low-level engagement data to develop better models of the learning objects being interacted with. Douglas, Bermel, Alam, and Madhavan develop a model of learner engagement in a MOOC, suggesting that closer analysis of engagement and 'success' or longevity indicates different behaviours between the engagement groups. Similarly, Dvorak and Jia discuss the relationship between engagement and academic performance, characterising engagement in terms of online work habits, measured through timeliness, regularity, and intensity of work. The authors demonstrated that higher grades are associated with earlier and more regular work on assignments.

Taking a different approach to enriching analysis of student interaction with learning-objects, Waddington, Nam, Lonn, and Teasley investigate the addition of learning-resource category data to early warning systems. The authors observed that students who use more resources in the 'exam preparation' category are more likely to receive a final grade of B or above. Applying this richer perspective to specific subject-based learning activities, Sutherland and White, and Vieira, Goldstein, Purzer, and Magana discuss learning analytics for algebra learning, and experimentation strategies in engineering design, respectively. Sutherland and White's paper indicates that log-data regarding student algebraic formulations can be used to identify strategies that are related to outcomes. Similarly, Vieira, et al., discuss the strategies students adopt while conducting experiments in engineering design. They maintain that log-data from a Computer-Aided Design (CAD) platform can be used to identify student strategies, and relate these to outcome. There is strong potential of learning analytics research for understanding the choices that students make in such assessment tasks (Schwartz \& Arena, 2013).

Finally, Howley and Rosé discuss the complex interactions of theory and methods in computational modeling of group learning processes. Discussing a body of work emerging from their research, they describe the validation across contexts of machine learning approaches to group learning processes. 
(2016). Learning analytics: Richer perspectives across stakeholders. Journal of Learning Analytics, 3(3), 1-4. http://dx.doi.org/10.18608/jla.2016.33.1

Analyses across the examined papers indicate the importance of learning design and capturing of learning data at the appropriate level, in developing and deploying learning analytics approaches.

\section{LEARNING LEARNING ANALYTICS: SPECIAL SECTION ON LEARNING ANALYTICS TUTORIALS}

This issue also brings a special section comprising five tutorials that discuss different methods and approaches used in learning analytics. Learning analytics is frequently referred to as bricolage field that builds on the methods from many different research fields. As such, learning analytics must draw its methods from its constituent fields, prompting a need for resources to share and teach these methods across an interdisciplinary audience. To address this gap in the use of different methods, members of the learning analytics community have been organizing numerous workshops and tutorials at the central events of the Society for Learning Analytics Research (SoLAR):the International Conference on Learning Analytics and Knowledge (LAK) and the Learning Analytics Summer Institute (LASI). In this issue, Dragan Gašević and Mykola Pechenizkiy bring a compilation of five selected tutorials presented at LAK 14 and LASI 14. These tutorials cover a wide range of methods including, epistemic network analysis, social network analysis, text analysis with Co-Metrix, microgenetic analysis of learning with $\mathrm{R}$, and user centered design of visualizations for learning analytics. We hope that this special section will help those working in the field of learning analytics to embrace new analytic methods in their research and practice. We would also like to invite prospective authors to contact the JLA editorial team with new ideas for writing tutorial papers for consideration in the journal, to continue this critical activity for developing the field.

We would also like to note that this special section is another example of successful collaboration between the sisters' societies - SoLAR and the International Educational Data Mining Society (IEDMS) as the special section is edited by the current presidents of the societies, who jointly chaired the program of LASI' 14 .

\section{$4 \quad$ EDITORIAL TEAM UPDATE}

In our last editorial we introduced the new international editorial team. They are now taking on more of the day-to-day tasks of running and developing the journal with the guidance of the established editors.

As part of these changes, the editorial board of the Journal of Learning Analytics has been reviewing the scope and framing of the 'Hot Spots' section. The Hot Spots section serves two purposes for the SoLAR community: first, the section broadens the readership and impact of the journal, through reaching out to the practitioner community; and second, it broadens the evidence base of the journal for both practitioners and researchers through the publication of practitioner-oriented research. The section aims to provide timely, practical reports of relevance to both practitioners and researchers in the SoLAR community. 
(2016). Learning analytics: Richer perspectives across stakeholders. Journal of Learning Analytics, 3(3), 1-4. http://dx.doi.org/10.18608/jla.2016.33.1

Our initial step was to make it easier to find previous Hot Spots articles via search (and a direct link within the editorial policies page). In fact, keywords have now been added as metadata across all journal articles, which should improve the quality of navigation for both new submissions and published papers. We are actively exploring other options to improve the structure of articles in the journal for our diverse audiences.

To gather opinions and suggestions for reframing the Hot Spots section, we conducted online consultations within the editorial board and beyond, and we thank everyone who participated. Overwhelmingly consultation respondents were supportive of new types of submissions to the journal including technical reports, resources such as dataset descriptions, short papers, and responses or commentaries. Such submission types are not uncommon across the disciplinary fields learning analytics is grounded in, and the editors intend to take the consultation feedback into consideration in revising the section policies of the journal. However, we are also mindful of the concern to maintain high scholarly standards in the journal, and the associated need to distinguish clearly between papers undergoing different peer review models. By drawing on practices in other respected venues, we hope to continue developing the journal's impact as the venue of choice for a rich spectrum of learning analytics research, which is accessible and relevant to stakeholders across academia, education more broadly, and industry.

\section{REFERENCES}

Clow, D. (2012). The learning analytics cycle: closing the loop effectively. Proceedings of the 2nd International Conference on Learning Analytics and Knowledge (LAK'12), 134-138. https://dx.doi.org/10.1145/2330601.2330636

McPherson, J., Tong, H. L., Fatt, J. S., \& Liu, D. Y. T. (2016). Student perspectives on data provision and use: starting to unpack disciplinary differences. Proceedings of the 6th International ACM Conference on Learning Analytics and Knowledge (LAK'16), 158-167. https://dx.doi.org/10.1145/2883851.2883945

Schwartz, D. L., \& Arena, D. (2013). Measuring what matters most: Choice-based assessments for the digital age. Boston Massachusetts: MIT Press.

Wise, A. F., Vytasek, J. M., Hausknecht, S., \& Zhao, Y. (2016). Developing learning analytics design knowledge in the "middle space": The student tuning model and align design framework for learning analytics use. Online Learning, 20(2). Retrieved from http://olj.onlinelearningconsortium.org/index.php/olj/article/view/783 